\title{
Perfil funcional básico de los jóvenes del programa nacional de tecnificación en bádminton
}

\author{
Basic Functional Assessment of Young Players from the \\ National Badminton Sports Training Plan
}

\author{
María Isabel Gil-López* \\ Cristina Sanchez-De la Vieja \\ Raquel Hernández-García 1,2 (1)
}

1. Department of Physical Activity and Sport, Faculty of Sports Sciences, Regional Campus of International Excellence "Campus Mare Nostrum", Universidad de Murcia, 30720 San Javier, España.

2. Sports and Musculoskeletal System Research Group (RAQUIS), Universidad de Murcia, 30100 C.P., España.

\begin{abstract}
Resumen
El objetivo del presente estudio fue analizar el perfil funcional de un grupo de jóvenes deportistas, todos pertenecientes al programa nacional de tecnificación en bádminton. Se evaluaron a 43 jugadores de bádminton (26 chicos y 17 chicas), de entre 15 y 16 años, con una experiencia mínima de 3 años de práctica competitiva. Se evaluaron los patrones motores básicos utilizando para ello, la batería de valoración funcional "Basic Functional Assessment" compuesta por cinco pruebas funcionales: Overhead Squat (OHS), Hurdle Step (HS), Forward Step Down (FSD), Shoulder Mobility (SM) y Active Straight Leg Raise (ASLR). Los resultados muestran que el grupo de chicos ha presentado una mayor incidencia de compensaciones totales que el grupo de chicas. La compensación que más se ha repetido en toda la muestra ha sido la protracción cervical, vista en la prueba SM; seguida de la pérdida de flexión de hombros, en el test OHS. De manera conjunta como equipo de bádminton sin diferenciación de sexo obtuvieron una media de 20.86 compensaciones sobre 75 . Las compensaciones que más han presentado los jugadores se encontraban en el complejo de la cadera, en el complejo del hombro, así como una falta de estabilidad del core.
\end{abstract}

Palabras clave: Valoración funcional básica, calidad del movimiento, riesgo de lesión, prevención, bádminton.

\begin{abstract}
The main of this study was to analyze the Functional profile of a group of young athletes, all of them belonging to the national badminton technification programme. 43 badminton players ( 26 boys and 17 girls) between the age of 15 and 16 were evaluated with a minimum experience of 3 years of competitive practice. Basic motor patterns were evaluated using the "Basic Functional Assessment" Functional rating battery consisting of five Functional tests: Overhead Squat (OHS), Hurdle Step (HS), Forward Step Down (FSD), Shoulder Mobility (SM) y Active Straight Leg Raise (ASLR). The results show that the boys' group has had a higher incidence of total compensation than the girls' group. The most repeated compensation throughout the sample has been cervical protraction, seen in the SM test; followed by the loss of shoulder flexion, in the OHS test. Together as a team of badminton without gender differentiation, they obtained an average of 20.86 compensations over 75 . The compensations that players have presented the most were in the hip complex, in the shoulder complex, as well as a lack of core stability.
\end{abstract}

Keywords: Basic functional assessment, movement quality, injury risk, prevention, badminton.

\section{* Autor de correspondencia: María Isabel Gil-López, mariaisabel.gil1@um.es}

Recibido: 07 de octubre de 2020

Aceptado: 24 de noviembre de 2020

Publicado: 31 de enero de 2021

Como citar (APA): Gil-López, M., Sanchez-De la Vieja, C. \& Hernández-García, R. (2021). Perfil funcional básico de los jóvenes del programa nacional de tecnificación en bádminton. JUMP, (3), 1-9. https://doi.org/10.17561/jump.n3.1

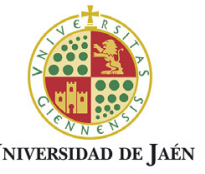




\section{Introducción}

En España es muy común la iniciación deportiva en etapa escolar, con la posibilidad de que los jóvenes continúen su formación con un proceso de tecnificación avalado por el Consejo Superior de Deportes (CSD). Este trabajo se centrará en analizar las demandas del bádminton, y más específicamente las adaptaciones funcionales provocadas en este deporte en la etapa de tecnificación.

El bádminton es un deporte de raqueta, se juega en una cancha dividida por una red, puede ser jugado en modalidad individual (1 vs. 1) o dobles (2 vs. 2) (Guillain, 2002). El principal objetivo es pasar el volante por encima de la red, cayendo este dentro del campo contrario, para ello, se busca enviar el volante a zonas que dificulten su devolución (Cabello, 2000). Se trata de un deporte muy exigente e intenso, donde hay que mantener un alto estado de concentración; la duración de las jugadas suele ser corta (en la élite es de alrededor 6-8 segundos) y de muy alta intensidad que se intercalan con cortos períodos de descanso (alrededor de 15 segundos) (Phomsoupha y Laffaye, 2015; Raman y Nageswaran, 2013). A este estado de concentración se suma la intensidad de los continuos cambios de dirección, saltos, desplazamientos agresivos hacia la red y movimientos rápidos de los brazos desde una variedad de posiciones (Phomsoupha y Laffaye, 2015).

Aunque se trate de un deporte sin contacto, existe un riesgo significativo de lesiones, con una incidencia del $44-66 \%$ de lesiones en jugadores de bádminton (Herbaut et al., 2018). La mayoría de autores involucran la extremidad inferior como la más lesionada (Herbaut et al., 2018; Herbaut y Delamoy 2012; Jacobson et al., 2005; Jeyaraman et al., 2012; Kimura et al., 2010; Revees et al., 2015).

El estudio de Reeves et al., (2015) sobre lesiones en bádminton estableció que, un 34.4\% de las lesiones fueron ocasionadas por movimientos que implican girar; el $22.5 \%$ se atribuye a los movimientos generales como correr; un $10.9 \%$ a los movimientos específicos del bádminton; $7.3 \%$ al aterrizaje en los saltos. Otros autores establecen que las lesiones de las extremidades inferiores en jugadores de bádminton generalmente ocurren durante el aterrizaje del salto (Kimura et al., 2010). Herbaut et al., (2018) también señalan la recepción del salto como el desencadenante de lesiones, en su estudio representan que el 33\% al 49\% de las lesiones fueron esguinces de tobillo y que la mayoría ocurrieron después del salto. Por otro lado, el aumento de la fatiga altera la biomecánica de los jugadores, lo que puede causar un mayor riesgo de sufrir una lesión de tobillo (Herbaut y Delannoy 2020). La fatiga influye en la forma en que se recepciona el salto haciendo que los tendones sean menos potentes y más inestables. Probablemente el componente excéntrico del bádminton y los movimientos rápidos y explosivos que realizan los jugadores durante el juego hagan que se sobrecarguen los tendones de rodillas y tobillos (Jeyaraman et al., 2012). También existen movimientos específicos del bádminton, como es el lunge, este requiere un alto nivel de dinámica y estabilidad de las extremidades inferiores para poder realizar una correcta ejecución del movimiento de la raqueta (Huang et al., 2014). La acción repentina de detener y avanzar en pasos de lunge podría producir cargas extenuantes en las articulaciones de las extremidades inferiores (Hu et al., 2015). El uso de pasos de lunge están estrechamente relacionados con la habilidad, el éxito de la competición y, a menudo, con mayor riesgo de lesiones (Dos Santos et al., 2018). También aparecen lesiones en el brazo y en el hombro, pero en ocasiones las atribuyen a una técnica deficiente, sin embargo, las lesiones en piernas y espalda se atribuyen más a menudo a una falta de fuerza o movilidad (Phomsoupha y Laffaye 2020).

La prevención de lesiones en atletas juveniles es esencial no solo para mantener su carrera deportiva competitiva sino también para evitar otras complicaciones que puedan afectar su calidad de vida a largo plazo (Ardern et al., 2018). Por ello, se considera necesario incluir estrategias para la valoración del riesgo de lesión en la etapa juvenil.

Stodden et al., (2008) desarrollaron un modelo conceptual y postularon que, en la infancia media y tardía, la competencia motriz, a través de las habilidades fundamentales del movimiento (HFM), han contribuido al desarrollo físico, cognitivo y social de los niños y se cree que proporciona la base para un estilo de vida activo. La justificación para promover el desarrollo de HFM en la infancia se basa en la existencia de evidencia sobre los beneficios actuales o futuros asociados con la adquisición de HFM (Lubans et al., 2010). Mediante la evaluación de las HFM a través de pruebas 
funcionales, se puede examinar la sincronía del control neuromuscular, el rango de movimiento, la fuerza, la resistencia, el equilibrio y la coordinación necesaria para completar los movimientos (Kivlan, 2012).

La evaluación funcional en deportistas está siendo una herramienta clave en la reducción del riesgo de lesión. Para ello, se emplean pruebas o tests con el objetivo de detectar movimientos compensatorios producidos por una limitación en los patrones de movimiento, que suelen aparecer como fruto de ciertos déficits y asimetrías (Bennett et al., 2019). De las herramientas más utilizadas para este fin, destacan dos principalmente: FMS y BFA (Basic Functional Assessment). FMS Consta de siete pruebas diseñadas para detectar patrones motores débiles (Minick et al., 2010). Es una de las herramientas más utilizadas para evaluación funcional, sin embargo, genera oposición entre autores respecto a su validez para predecir el riesgo de lesión (Moran, 2017). BFA es una batería de cinco pruebas diseñadas para evaluar la calidad de movimiento durante la ejecución de patrones fundamentales del movimiento, mediante el análisis de compensaciones 0 disfunciones manifestadas en todo tipo de población (Hernandez-García et al., 2020a), ya que los patrones fundamentales se establecen como la base y esencia de movimientos más complejos (Hulteen et al., 2018)

En este sentido el objetivo del presente estudio fue describir el perfil funcional básico de jugadores juveniles de bádminton a través de las compensaciones que se observan en la ejecución de patrones motores mediante la batería BFA.

\section{Material y Método}

El presente estudio se desarrolló por medio de un convenio de colaboración entre la Federación Española de Bádminton, la Universidad de Jaén y la Universidad de Murcia.

\section{Muestra}

Participaron un total de 43 jugadores de bádminton (26 chicos y 17 chicas), con edades comprendidas entre los 15 y 16 años, y con una experiencia mínima de 3 años de práctica competitiva. Participaron voluntariamente en este estudio. Todos ellos formaban parte del Proyecto Nacional de Tecnificación, en la categoría sub17 de la Federación Española de Bádminton.
Los criterios de inclusión fueron: a) pertenecer al grupo de tecnificación nacional; b) No tener ninguna lesión en el momento de la evaluación.

\section{Diseño y Procedimiento}

Se trata de un estudio descriptivo (Thomas et al., 2001) y se empleó la metodología observacional para valorar el perfil funcional básico. Para ello, se empleó el protocolo de la batería "Basic Functional Assessment" (Hernandez-Garcia et al., 2020a), creado para obtener información sobre la calidad del movimiento en patrones motores básicos, además de las compensaciones o disfunciones manifestadas.

La prueba fue realizada durante el año 2020, cuando se encontraban concentrados en Huelva. La valoración se implementó durante una única sesión en el campo de entrenamiento. Se les explicó detalladamente tanto a los jugadores como a los entrenadores/tutores en qué consistía la valoración, así como el funcionamiento que se llevará a cabo para su realización. Todos los jugadores participantes firmaron el consentimiento informado por parte de sus entrenadores/tutores al ser menores de edad.

Las pruebas fueron grabadas desde tres planos (sagital, frontal anterior y frontal posterior) según se requiere en cada una de las pruebas. Los vídeos fueron realizados con una cámara GoPro Hero7 con 4K de resolución de captura de vídeo. Se consideraron repeticiones nulas aquellas en las que los sujetos no reproducían el patrón que se le había indicado o bien cuando perdía la estabilidad, se caía o se desplazaba de su base de sustentación inicial durante la ejecución. El orden de las pruebas fue el mismo para cada deportista y con la intención de minimizar cualquier posible efecto negativo entre ellas. Las 5 pruebas utilizadas fueron: Overhead Squat (OHS), Hurdle Step (HS), Forward Step Down (FSD), Shoulder Mobility (SM) y Active Strength Leg Raise (ASLR).

Tras las grabaciones se procedió al análisis de los vídeos para establecer el perfil funcional de cada uno de los jugadores. Para ello, se empleó el programa de edición de vídeo Kinovea 0.8.15. Un único observador, estudiante de último curso del Grado en Ciencias de la Actividad Física y el Deporte, fue el encargado de observar los vídeos y analizarlos. El observador obtuvo una formación previa durante cuatro meses antes de la realización del estudio. 


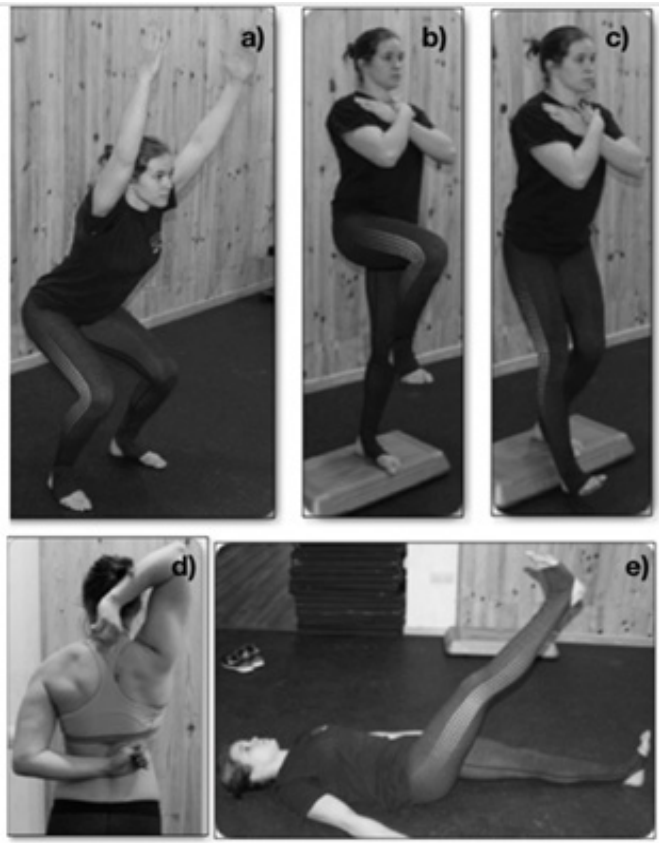

Imagen 1. Pruebas de la batería (Basic Functional Assessment". a) Overhead Squat; b) Hurdle Step; c) Forward Step Down; d) Shoulder Mobility; e) Active Strength Leg Raise.

Fuente: Hernandez-Garcia et al. (2019).

\section{Análisis estadístico}

Los datos fueron codificados y analizados con el paquete estadístico SPSS (versión 21.0 para Windows). Se realizó un análisis descriptivo de las variables. Se analizó la normalidad de los datos a través de la prueba Shapiro-Wilk, y se realizó la comprobación la homogeneidad de varianza mediante la prueba de Levene, con un nivel de significación p>.05. Después de este análisis, se realizó una comparación de medias para muestras independientes utilizando la prueba T de Student en el caso de variables paramétricas ( $p>$.05), y la prueba Mann Whitney $U$ para las pruebas no paramétricas $(p<.05)$. El valor de diferencia estadísticamente significativa entre grupos (masculino y femenino) se mostró siempre que $p<.05$.

\section{Resultados}

La tabla 1 muestra de manera descriptiva el número de compensaciones que se han dado en cada una de las pruebas según el sexo.

La prueba que ha presentado mayor número de compensaciones ha sido FSD tanto en

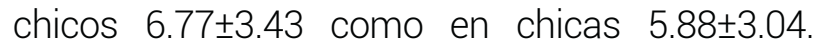
Para establecer un perfil funcional es necesario contar con los resultados de todas las pruebas de manera conjunta, de este modo se ha visto que el grupo de chicos tiene una media de $22.54 \pm 7.20$ compensaciones entre todas las pruebas, siendo ligeramente superior al resultado que encontramos en el grupo de chicas, con una media de 18.29 \pm 6.81 compensaciones entre todas las pruebas. Hay que tener en cuenta que el máximo número de compensaciones que se puede alcanzar es de 75. Ninguno de los dos grupos alcanza la mitad de las compensaciones por lo que muestran un perfil funcional óptimo. De manera conjunta como equipo de bádminton sin diferenciación de sexo obtuvieron una media de $20.86 \pm 7.28$ compensaciones sobre 75 , mostrando un perfil funcional óptimo.

Tabla 1. Compensaciones por prueba y género

\begin{tabular}{ccccc}
\hline Prueba & Grupo & N & Media & Desv.Tip \\
\hline \multirow{2}{*}{ OHS } & Masculino & 26 & 4.00 & 2.70 \\
& Femenino & 17 & 3.76 & 2.66 \\
HS & Masculino & 26 & 4.31 & 2.57 \\
& Femenino & 17 & 2.06 & 1.89 \\
FSD & Masculino & 26 & 6.77 & 3.43 \\
& Femenino & 17 & 5.88 & 3.04 \\
\multirow{3}{*}{ SM } & Masculino & 26 & 3.73 & 1.31 \\
& Femenino & 17 & 2.82 & 1.85 \\
ASLR & Masculino & 26 & 3.73 & 1.99 \\
& Femenino & 17 & 3.76 & 2.51 \\
\multirow{2}{*}{ Total } & Masculino & 26 & 22.54 & 7.20 \\
& Femenino & 17 & 18.29 & 6.81 \\
\hline
\end{tabular}

Nota: $\mathrm{OHS}=$ Overhead Squat; HS= Hurdle Step; FSD= Forward step Down; SM= Shoulder Mobility; ASLR= Active Strength Leg Raise; Desv.Tip= Desviación Típica.

En la figura 1 y 2, se muestra un gráfico con las compensaciones más frecuentes en toda la muestra y por sexo.

Las compensaciones que más se observaron en toda la muestra fueron, la protracción cervical en ambos lados en el SM, la perdida de flexión de hombros en el OHS, movimiento torácico en ambos lados en el FSD y la extensión lumbar en el ASLR tanto con pierna derecha como izquierda.

A la hora de distinguir por sexo se observa que en el grupo de chicos la compensación más común sigue siendo la protracción cervical durante la prueba SM, seguido de la pérdida de flexión de hombros en OHS. A diferencia, observamos en el grupo de chicas que la compensación más común ha sido la extensión lumbar durante la prueba ASLR, seguido del movimiento torácico durante la prueba FSD y la pérdida de flexión de hombros en $\mathrm{OHS}$. 


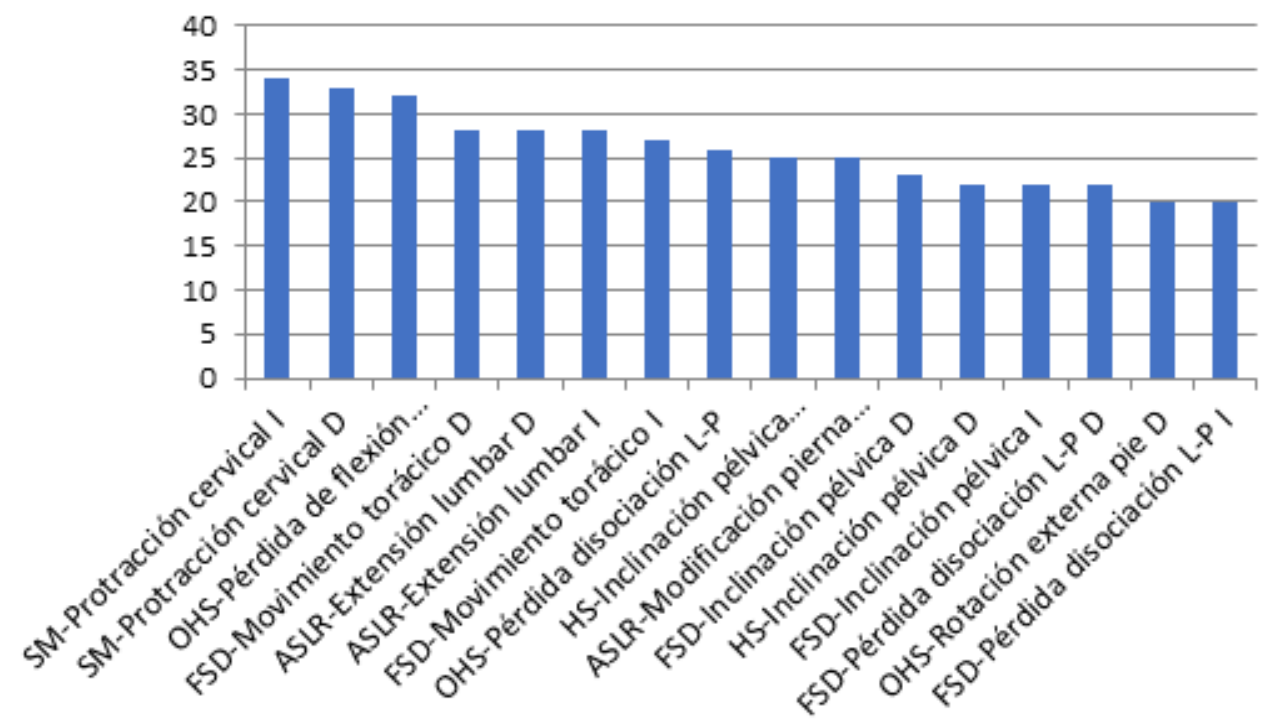

Figura 1. Compensaciones más frecuentes en toda la muestra

Nota: $\mathrm{OHS}=$ Overhead Squat; HS= Hurdle Step; FSD= Forward step Down; SM= Shoulder Mobility; ASLR= Active Strength Leg Raise; I= Izquierda; D= Derecha; L-P=Lumbo-Pélvica.

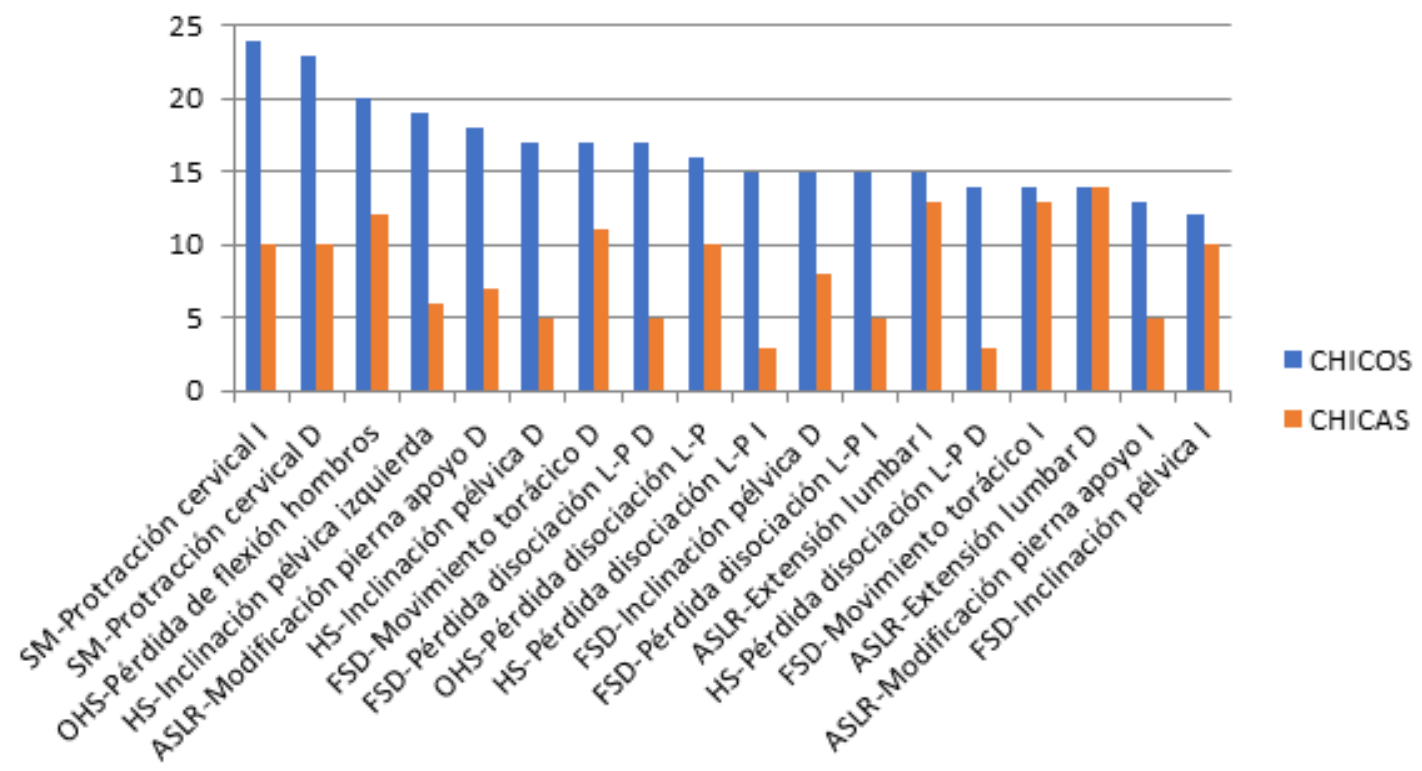

Figura 2. Compensaciones más frecuentes por género

Nota: $\mathrm{OHS}=$ Overhead Squat; HS= Hurdle Step; FSD= Forward step Down; SM= Shoulder Mobility; ASLR= Active Strength Leg Raise; I= Izquierda; $D=$ Derecha; L-P=Lumbo-Pélvica.

Las variables que asumen normalidad fueron tanto en compensaciones como en porcentajes del HS, FSD y en el SM ( $p>$.05).

Antes de realizar la comparación de medias se realizó la prueba Levene para comprobar la homogeneidad de las varianzas, en los resultados se encontró con un valor de p>.05 en todas las variables, por lo que existe una homogeneidad de varianza en la población de estudio, permitiendo la realización de comparación entre ambos grupos.

La única prueba que muestra diferencias estadísticamente significativas entre sexos es el número de compensaciones en el HS ( $p<.05)$. 
Tabla 2. Comparación de medias entre género

\begin{tabular}{lllccccc}
\hline & & & & \multicolumn{3}{c}{$\begin{array}{c}\text { 95\% Confidence } \\
\text { Interval }\end{array}$} \\
\cline { 6 - 8 } & & & & & Lower & Upper \\
\hline OHS & Student'st & 0.2812 & 41.0 & .780 & -1.4545 & 1.93 \\
HS & Mann- & 107 & & $.004 *$ & 1.000 & 4.00 \\
& Whitney U & & & & & \\
FSD & Student'st & 0.8656 & 41.0 & .392 & -1.1822 & 2.96 \\
SM & Student'st & 1.8854 & 41.0 & .066 & -0.0646 & 1.88 \\
ASLR & Student'st & -0.0492 & 41.0 & .961 & -1.4258 & 1.36 \\
Total & Student'st & 1.9301 & 41.0 & .061 & -0.1967 & 8.69 \\
\hline
\end{tabular}

Nota: $\mathrm{OHS}=$ Overhead Squat; HS= Hurdle Step; FSD= Forward Step Down; SM= Shoulder Mobility; ASLR= Active Strength Leg Raise.

\section{Discusión}

El perfil funcional de los jugadores de bádminton se sitúa en valores adecuados, ya que poseen una media porcentual de $26.83 \% \pm 8.57 \%$ y $21.78 \%$ $\pm 8.10 \%$ para chicos y chicas respectivamente. Esto puede indicar que la calidad de movimiento en los patrones motores básicos evaluados es adecuada si se compara por ejemplo con otros estudios que también emplearon la batería BFA; Gil-López et al., (2018) mostraron porcentajes de 45.74\% \pm 2.01 en un grupo de judokas; HernándezGarcía et al., (2020b) indicaron un porcentaje de $65.79 \% \pm 11.80 \%$ compensaciones en jugadoras profesionales de fútbol.

Si analizamos cada una de las pruebas encontramos que, en la prueba OHS la compensación más común en ambos sexos fue la perdida de la flexión de hombros. Esto se puede dar por una falta de activación de la porción media y baja del trapecio, romboides y manguito rotador, así como una sobre activación del dorsal ancho, pectoral mayor y redondo mayor (Bishop et al., 2016). Otras compensaciones que también destacaron en la realización de la prueba fueron la pérdida de disociación lumbo pélvica y la rotación externa del pie, coincidiendo con Gil-López et al. (2018) y Hernández-García et al., (2020b). La rotación externa de pies podría deberse a una falta de dorsiflexión de tobillo (Bishop et al., 2016) o bien a la hiperactivación de los rotadores externos de cadera en comparación con los rotadores internos (Hides et al., 2016). La falta de dorsiflexión de tobillo puede impedir realizar una sentadilla profunda al detener prematuramente el movimiento hacia delante de la tibia. Esto puede requerir un mayor balance compensatorio posterior del fémur para completar la sentadilla, que probablemente haga que aumente la dificultad por el desplazamiento posterior del centro de masas (Rabin y Kozol, 2017). La pérdida de disociación lumbo-pélvica se relaciona con una falta de equilibrio entre rotadores externos e internos de la cadera, provocando un déficit de estabilidad lumbo-pélvica ocasionando dolor en la zona lumbar y menor estabilidad de la musculatura del tronco (Hides et al., 2016). La activación de la musculatura del tronco es esencial para el control lumbo-pélvico (Shin et al., 2017). Esta musculatura cumple una función importante a la hora de la estabilidad lumbo-pélvica, si no se activa o hay una mala activación y/o coordinación de la musculatura del tronco, se crearán patrones compensatorios que darán como resultado un aumento del riesgo de lesión y sobrecargas (De Blaiser et al., 2018).

Aparece una marcada diferencia entre sexos en la prueba HS, siendo superior en chicos, mostrando una diferencia estadísticamente significativa con valor $p<.05$. Estos datos coinciden con los encontrados en un estudio con atletas de crossfit, donde Hernández-García y Toledo (2019) vieron diferencias entre sexos principalmente en el apoyo monopodal bajo carga. Dentro de las compensaciones con mayor presencia en HS, destacar la inclinación pélvica presente en ambos sexos, con bastante más incidencia en chicos. Esta inclinación pélvica, se podría dar por un conjunto de disfunciones, como son, una hiperactivación del cuadrado lumbar, junto a una eversión del pie de apoyo, que a su vez se relaciona con una falta de estabilidad en la articulación subastragalina (Hernández-Garcia et al., 2020b).

En el análisis de FSD fue donde los deportistas mostraron mayor número de compensaciones, esta prueba se realiza de manera unipodal bajo carga mostrando los déficits por movilidad, estabilidad y de capacidad. Las compensaciones que se dieron con mayor frecuencia durante esta prueba en toda la muestra fueron, la inclinación pélvica derecha/izquierda; movimiento torácico derecha/izquierda; y pérdida de disociación lumbo-pélvica derecha/izquierda. Los sujetos que muestran una peor calidad de movimiento, durante un apoyo monopodal parecen tener menos fuerza abductora de cadera y cuádriceps y una menor flexibilidad en la banda iliotibial / tensor de la fascia lata en comparación con sujetos que tienen una buena calidad de movimiento 
(Park et al., 2013). Hernández-García et al., (2020b) vieron que, en la prueba HS y FSD la inclinación pélvica, la pérdida de disociación lumbo-pélvica y el movimiento torácico fueron las manifestaciones más significativas. Estas compensaciones se pueden dar por un déficit en el core. En nadadores federados que no realizaban un entrenamiento del componente central del cuerpo se vio que tenían más probabilidad de sufrir una lesión y además no obtenían resultados satisfactorios en los test de evaluación de la estabilidad central (García, 2017). La falta de estabilidad del core se relaciona con lesiones de los miembros inferiores también en población deportista sana, pudiendo ser un factor de riesgo y antecesor de una lesión (De Blaiser et al., 2018).

Las compensaciones encontradas en la prueba SM coindicen con los resultados encontrados en Gil-López et al., (2018); Hernández-García et al., (2020b); Hernández-García y Toledo, (2019). SM es una prueba de movilidad y estabilidad de hombros, y en bádminton se precisa de forma significativa estas capacidades para reducir el riesgo de lesión y poder jugar en el alto nivel. Las compensaciones que más aparecieron en el SM fueron la protracción cervical derecha/izquierda. Tal como vieron Castropil y Arnoni (2014), donde en una postura con la cabeza adelantada se vio en más de la mitad de los judocas evaluados. Deficiencias en la cadena cinética por donde se transfieren las fuerzas; en el core, la columna, la cadera, el rango de movimiento glenohumeral y la cinemática escapular pueden ser un precedente a una patología de hombro (Andersson et al., 2017).

En cuanto a los resultados obtenidos en la prueba ASLR, fue la extensión lumbar la compensación más común, Sjödahl et al., (2016) vieron que los patrones de activación motora alterados influyen en la capacidad de las mujeres para estabilizar la pelvis durante el ASLR. La extensión lumbar se puede relacionar con una falta de la extensibilidad o cortedad de la musculatura isquiosural. Siguiendo a Cejudo et al., (2019) vieron en jugadores de fútbol que el rango de movimiento (ROM) va disminuyendo con la edad y esto podría llevar a los jugadores a patrones de movimiento compensatorios y aumentar el riesgo lesión en las articulaciones involucradas. Cabe destacar que una falta de eficiencia y asimetrías en ASLR se ha visto relacionado con mayores deficiencias y asimetrías en el rendimiento de tareas globales como el cambio de dirección en mujeres deportistas (Lockie et al., 2015).

En cuanto al perfil funcional global de todos los jugadores evaluados es considerado aceptable ya que han obtenido una media de $40.83 \pm 14.01$ compensaciones sobre 75 siendo este el total posible. Las compensaciones que más se han manifestado están relacionadas con falta de estabilidad en la extremidad inferior y el tronco. La mayoría de los autores involucran la extremidad inferior como la más lesionada (Herbaut et al., 2018; Jacobson et al., 2005; Reeves et al., 2015).

\section{Limitaciones y propuestas de investigaciones futuras}

Las principales limitaciones del presente estudio radican en la falta de datos de la muestra tales el peso, la talla, los años de experiencia en competición, los resultados, entre otros. Otra limitación es el pequeño número muestral, sería interesante incrementar el número de la muestra y obtener unos resultados con mayor alcance. Así como la posibilidad de haber evaluado algunas pruebas analíticas, y/o de fuerza para relacionarlas con los datos obtenidos en la funcionalidad.

Así, sería interesante establecer una relación de los resultados obtenidos con las horas de entrenamiento semanal, y las lesiones ocurridas durante una temporada, de este modo se podría determinar que BFA no solo es una batería que nos aporta información sobre déficit en los patrones motores, sino que, también nos puede dar información como predictor de futuras lesiones.

\section{Conclusiones}

El perfil funcional de los jugadores de bádminton sub-17 se considera adecuado, con una media total de $20.86 \pm 7.28$ compensaciones sobre 75 siendo este el máximo. Las compensaciones que más han presentado los jugadores se encontraban en el complejo de la cadera, inestabilidad en articulaciones que implican el complejo articular del hombro, así como una falta de estabilidad del core. Solo en la prueba HS se encontraron diferencias estadísticamente significativas entre sexos, siendo mayor el número de compensaciones mostradas en chicos que en chicas. 


\section{Aplicaciones prácticas}

La muestra de estudio son jóvenes deportistas con proyección a alto rendimiento y este test es válido para el establecimiento de su perfil funcional básico. Podría ser una estrategia para identificar a los jugadores con limitaciones en los patrones motores al comienzo de la temporada deportiva.

De este modo resulta más fácil individualizar partes de los entrenamientos y llevar a cabo un ejercicio correctivo con cada jugador para corregir los déficits funcionales que presente. Teniendo en cuenta los resultados del estudio, y que la mayoría de las deficiencias funcionales se encuentran en el complejo lumbo-pélvico, incluiremos ejercicios previos a la parte principal del entrenamiento enfocados a mejorar la funcionalidad de la cadera, ya sea a través de ejercicios de movilidad o ejercicios de activación, como puede ser el glute bridge para la activación del glúteo, o ejercicios de inhibición para aquellos músculos que se encuentres más rígidos, como puede ser el uso del foam roller para la musculatura isquiosural.

Con el sistema de evaluación empleado, tanto entrenadores como preparadores físicos, tienen una fuerte herramienta para trabajar en la reducción del riesgo de lesión.

\section{Financiación}

Este trabajo ha sido ha sido financiado por Badminton World Federation (BWF), bajo la convocatoria Research_Grant_2018 (2018/00465/001).

\section{Referencias}

Andersson, S. H., Bahr, R., Clarsen, B., \& Myklebust, G. (2017). Preventing overuse shoulder injuries among throwing athletes: a cluster-randomised controlled trial in 660 elite handball players. British Journal of Sports Medicine, 51(14), 1073-1080.

Ardern, C. L., Ekas, G. R., Grindem, H., Moksnes, H., Anderson, A. F., Chotel, F. \& Engebretsen, L. (2018). 2018 international olympic committee consensus statement on prevention, diagnosis and management of paediatric anterior cruciate ligament (ACL) injuries. British Journal of Sports Medicine, 52, 422-438. https://doi.org/10.1007/s00167-018-4865-y

Bennett, H., Davison, K., Arnold, J., Martin, M., Wood, S., \& Norton, K. (2019). Reliability of a movement quality assessment tool to guide exercise prescription (MovementSCREEN). International Journal of Sports Physical Therapy, 14(3), 424. https://doi.org/10.26603/ijspt2019024

Bishop, C., Edwards, M., \& Turner, A. N. (2016). Screening movement dysfunctions using the overhead squat. Professional Strength \& Conditioning, 42, 22-30.

Bishop, C., Villiere, A., \& Turner, A. (2016). Addressing movement patterns by using the overhead squat. Professional Strength \& Conditioning, 40, 7-12.

Cabello Manrique, D. (2000). Análisis de las características del juego en el bádminton de competición. Su aplicación al entrenamiento. Tesis Doctorales, (15). Recuperado de https:// www.cafyd.com/REVISTA/ojs/index.php/bbddcafyd/article/ view/123

Castropil, W., \& Arnoni, C. (2014). Postural patterns and adaptations in judo athletes. Archives of Budo, 10, 23-28.

Cejudo, A., Robles-Palazón, F. J., Ayala, F., Croix, M. D. S., OrtegaToro, E., Santonja-Medina, F., \& de Baranda, P. S. (2019). Agerelated differences in flexibility in soccer players $8-19$ years old. PeerJ, 7, e6236. https://doi.org/10.7717/peerj.6236

De Blaiser, C., Roosen, P. Willems, T., Danneels, L., Vanden, L., \& De Ridder, R. (2018). Is core stability a risk factor for lower extremity injuries in an athletic population? A systematic review. Physical Therapy in Sport, 30, 48-56

Dos'Santos, T., Thomas, C., Comfort, P., \& Jones, P. A. (2018). The effect of angle and velocity on change of direction biomechanics: An angle-velocity trade-off. Sports Medicine, 48(10), 2235-2253. https://doi.org/10.1007/s40279-0180968-3

García, M. (2017). La estabilidad del core y la prevención de lesiones en natación. http://redi.ufasta.edu.ar:8080/xmlui/ handle/123456789/1313

Gil-López, M., Garcia-Huertado., \& Hernandez-García, R. (2018). Valoración funcional básica del Judoka: un estudio piloto. Revista de artes marciales asiáticas, 13(2s), 20-22. https://doi. org/10.18002/rama.v13i2s.5500.

Guillain, J. Y. (2002). Histoire du badminton: du jeu de volant au sport olympique. Editions Publibook.

Herbaut, A., \& Delannoy, J. (2020). Fatigue increases ankle sprain risk in badminton players: A biomechanical study. Journal of Sports Sciences, 1-6, 1560-1565. https://doi.org/10.1080/026 40414.2020.1748337

Herbaut, A., Delannoy, J., \& Foissac, M. (2018). Injuries in French and Chinese regular badminton players. Science \& Sports, 33(3), 145-151. https://doi.org/10.1016/j.scispo.2018.02.001

Hernández-García, R., Aparicio-Sarmiento, A., Palao, J.M., y de Baranda, P. S. (2020b). Influencia de las lesiones previas en los patrones fundamentales del movimiento en jugadoras profesional de fútbol. RICYDE. Revista internacional de Ciencias del Deporte, 16(60), 214235.

Hernández-García, R., Gil-López, M., Martínez-Pozo, D., MartínezRomero, M., Aparicio-Sarmiento, A., Cejudo, A., Sainz de Baranda, P., \& Bishop, C. (2020a). Validez y fiabilidad del nuevo protocolo básico de evaluación funcional (BFA). International Journal of Environmental Research and Public Health, 17(13), 4845 .

Hernández-García, R., Toledo, D.; Fernández-García, I., \& TorresLuque, G. (2019). Valoración del perfil funcional en atletas de CrossFit. Estudio Piloto. Acción Motriz, 23, 100-108.

Hides, J. A., Oostenbroek, T., Franettovich Smith, M. M., \& Mendis, M. D. (2016). The effect of low back pain on trunk muscle size/function and hip strength in elite football (soccer) players. Journal of Sports Sciences, 34(24), 2303-2311. https://doi.org/10.1080/02640414.2016.1221526

Hu, X., Li, J. X., Hong, Y., \& Wang, L. (2015). Characteristics of plantar loads in maximum forward lunge tasks in badminton. PloS one, 10(9), e0137558. https://doi.org/10.1371/journal. pone.0137558

Huang, M. T., Lee, H. H., Lin, C. F., Tsai, Y. J., \& Liao, J. C. (2014). How does knee pain a ect trunk and knee motion during badminton forehand lunges? Journal of Sports Sciences, 32, 690-700.

Hulteen, R.M., Morgan, P.J., Barnett, L.M., Stodden, D.F., Lubans, D.R. (2018). Development of Foundational Movement Skills: A Conceptual Model for Physical Activity Across the Lifespan. 
Sports Medicine, 48, 1533-1540. https://doi.org/10.1007/ s40279-018-0892-6

Jacobson, J. A., Miller, B. S., \& Morag, Y. (2005). Golf and racquet sports injuries. Seminars in Musculoskeletal Radiology, 9, 346359.

Jeyaraman, R., District, E., \& Nadu, T. (2012). Prediction of playing anility in badminton from selected anthropometrical physical and physiological characteristics among inter collegiate players. International Journal of Advanced Research and Innovation, 2(3), 11.

Kimura,Y., Ishibashi,Y., Tsuda, E.,Yamamoto,Y.,Tsukada, H.,\& Toh, S.(2010). Mechanismsfor anterior cruciate ligament injuries in badminton. British Journal of Sports Medicine, 44, 1124- 1127.

Kivlan, B.R. (2012). Functional Performance Testing of the Hip in Athletes. International Journal of Sports Physical Therapy, 7(4), 402-412.

Lockie, R., Schultz, A., Callaghan, S., Jordan, C., Luczo, T., \& Jeffriess, M. (2015). A preliminary investigation into the relationship between functional movement screen scores and athletic physical performance in female team sport athletes. Biology of Sport, 32(1), 41-51. https://doi. org/10.5604/20831862.1127281

Lubans, D. R., Morgan, P. J., Cliff, D. P., Barnett, L. M., y Okely, A. D. (2010). Habilidades fundamentales de movimiento en niños y adolescentes. Medicina deportiva, 40(12), 1019-1035.

Minick, K. I., Kiesel, K. B., Burton, L. E. E., Taylor, A., Plisky, P., \& Butler, R. J. (2010). Interrater reliability of the functional movement screen. The Journal of Strength \& Conditioning Research, 24(2), 479-486. http://dx.doi.org/10.1136/bjsports-2016-096938

Moran, R.W., Schneiders, A.G., Mason, J., \& Sullivan, S.J. (2017). Do Functional Movement Screen (FMS) composite scores predict subsequent injury? A systematic review with meta-analysis. British Journal of Sports Medicine, 51, 1661 LP - 1669.

Park, K. M., Cynn, H. S., \& Choung, S. D. (2013). Musculoskeletal predictors of movement quality for the forward step-down test in asymptomatic women. Journal of Orthopaedic \& Sports Physical Therapy, 43(7), 504-510. https://www.jospt.org/ doi/10.2519/jospt.2013.4073
Phomsoupha, M., \& Laffaye, G. (2015). The science of badminton: game characteristics, anthropometry, physiology, visual fitness and biomechanics. Sports Medicine, 45(4), 473-495. https://doi.org/10.1007/s40279-014-0287-2

Rabin, A., \& Kozol, Z. (2017). Utility of the overhead squat and forward arm squat in screening for limited ankle dorsiflexion. The Journal of Strength \& Conditioning Research, 31(5), 12511258. https://doi.org/10.1519/JSC.0000000000001580.

Reeves, J., Hume, P. A., Gianotti, S., Wilson, B., \& Ikeda, E. (2015). A retrospective review from 2006 to 2011 of lower extremity injuries in badminton in New Zealand. Sports, 3(2), 77-86. https://doi.org/10.3390/sports3020077

Shin, S. H., Kang, S. R., Kwon, T. K., \& Yu, C. (2017). A study on trunk muscle activation patterns according to tilt angle during whole body tilts. Technology and Health Care: Official Journal of the European Society for Engineering and Medicine, 25(1), 73-81. https://doi.org/10.3233/THC-171308.

Sjödahl, J., Gutke, A., Ghaffari, G., Strömberg, T., \& Öberg, B. (2016). Response of the muscles in the pelvic floor and the lower lateral abdominal wall during the active straight leg raise in women with and without pelvic girdle pain: an experimental study. Clinical Biomechanics, 35, 49. https://doi.org/10.1016/j. clinbiomech.2016.04.007

Stodden, D. F., Goodway, J. D., Langendorfer, S. J., Roberton, M. A. Rudisill, M. E., Garcia, C., \& Garcia, L. E. (2008). A developmental perspective on the role of motor skill competence in physical activity: An emergent relationship. Quest, 60(2), 290-3. https://doi.org/10.1080/00336297.2008.10483582

Thomas, J. R., Nelson, J. K., Silverman, S. J., \& Silverman, S. J. (2001). Research Methods in Physical Activity 6th. Ed. Champaign, Illinois: Human Kinetics. 